Maltepe Journal of Mathematics

ISSN:2667-7660, URL:HTTP://DERGIPARK.ORG.TR/TR/PUB/MJM

Volume III Issue 2 (2021), PAGes 52-59. Doi:HtTPs://DOI.ORG/10.47087/MJM.883671

\title{
MORE ON SEMI QUOTIENT MAPPINGS AND SPACES
}

\author{
MUHAMMAD SIDDIQUE BOSAN \\ DEPARTMENT OF MATHEMATICS, KHWAJA FAREED UNIVERSITY OF \\ ENGINEERING AND INFORMATION TECHNOLOGY, RAHIM YAR KHAN, 64200, \\ PAKISTAN, +923038016521, ORCID ID: 0000-0002-9433-7960
}

\begin{abstract}
In our paper, semi quotient mappings and spaces properties are developed by the change of topology where the notion of semi quotient topology built the interest. Results of this article describe the more interest in our work with the contribution of extremally disconnected concept where the quotient space $J / N$ with this topology $s \tau_{Q}$ has surprisingly moved to an $s$-topological group.
\end{abstract}

\section{INTRODUCTION}

A mathematical discipline assembling the topology and group is called the topological group [8, 12. This discipline has very significant applications in almost all branches of natural sciences. In our arrangement operations of multiplicity and inverse on the continuity and its general forms will be discussed. The study of this weaker form of continuity with topological groups started in 1990s. Twenty-thirty years ago more interesting results relating to the discipline discussed in literature. In 2014, Bosan and Moiz [2] and [5] explored the notion of quasi $s$-topological groups, and quasi irresolute topological groups. We studied the concept of Levine [7] about topological spaces on semi open sets. Different mathematicians like Crossley et.al. 4 studied semi topological properties and Bohn 1] studied semi topological groups. Moreover, Siab et.al. 14 studied irresolute topological groups by using irresolute mappings. In continuation to these concepts Bosan et.al. 3, 5] studied classes of $s$-topological groups and $S$-topological groups. In 2016, Noreen et.al. introduced and defined semi quotient topology which is the generalization of quotient topology for spaces and groups [10. The motivation behind this work was to study the quotient topology by weakening the open set conditions and also explored semi quotient mappings stronger than semi continuous mappings, and then consider semi quotient spaces and groups [10.

We need also some basic information on a quotient group as: If $J$ is group and $H$ invariant subgroup, consider the collection $Q$ of all left cosets

2020 Mathematics Subject Classification. Primary: 54H11, 22A05, 54C08, 54H99.

Key words and phrases. semi continuous mapping, group structure, quasi topological group, semi quotient mapping.

(C)2019 Maltepe Journal of Mathematics.

Submitted on February 20 th. 2021. Published on October 30 th. 2021

Communicated by Ayhan ESI . 


$$
a H \text { of } H, a \in J .
$$

Define a multiplication in $Q$ as follows: For

$$
a H, b H \in Q \text {, we put } a H . b H=a b H .
$$

It is easy to verify that under this multiplication $Q$ is a group with $e H=H$ as the identity and $a^{\leftarrow} H$ as the inverse of $a H$ in $Q$. This group is called the quotient or factor group of $J$ by $H$ and it is denoted by $J / H$. $H$ is invariant subgroup of $J$. The mapping

$$
p: J \rightarrow J / H
$$

defined by $p(x)=x H$, for each $x \in J$ is called a natural projection.

In this paper, significant results with counter examples have also been proved. We have also used semi homeomorphism [6] and S-homeomorphism [13] changing a discipline to an other and support the discipline.

\section{PRELIMINARIES}

A mapping $\zeta: K \rightarrow M$ between topological spaces $K$ and $M$ is called:

- pre-semi-open 4 if for every semi-open set $A$ of $K$, the set $\zeta(A)$ is semiopen in $M$;

- $s$-open ( $s$-closed) if for every semi-open (semi-closed) set $A$ of $K$, the set $\zeta(A)$ is open (closed) in $M$;

- continuous if for each open set $V \subset M$ the set $\zeta^{\leftarrow}(V)$ is open in $K$.

- semi-continuous 77 (resp. irresolute [4]) if for each open (resp. semi-open) set $V \subset M$ the set $\zeta^{\leftarrow}(V)$ is semi-open in $K$. Equivalently, the mapping $\zeta$ is semi-continuous (irresolute) if for each $x \in K$ and for each open (semiopen) neighbourhood $V$ of $\zeta(x)$, there exists a semi-open neighbourhood $U$ of $x$ such that $\zeta(U) \subset V$;

- semi-homeomorphism [4, 6] if $\zeta$ is bijective, irresolute and pre-semi-open;

- $S$-homeomorphism [3] if $\zeta$ is bijective, semi-continuous and pre-semi-open".

- $S$-isomorphism if it is an algebraic isomorphism and topologically an $S$-homeomorphism,

- semi-isomorphism if it is an algebraic isomorphism and topologically a semihomeomorphism.

Definition 2.1. 1] An s-topological group is a group $(J, *)$ with a topology $\tau$ such that for each $x, y \in J$ and each neighbourhood $W$ of $x * y^{\leftarrow}$ there are semi open neighbourhoods $U$ of $x$ and $V$ of $y$ such that

$$
\mathrm{U} * \mathrm{~V}^{\leftarrow} \subset \mathrm{W} .
$$

Definition 2.2. [14 A triple $(\mathrm{J}, *, \tau)$ is an irresolute topological group with a group $(\mathrm{J}, *)$ and a topology $\tau$ such that for each $\mathrm{x}, \mathrm{y} \in \mathrm{J}$ and for each semi open neighbourhood $\mathrm{W}$ of $\mathrm{x} * \mathrm{y}^{\leftarrow}$, there exist semi-open neighbourhoods $\mathrm{U}$ of $\mathrm{x}$ and $\mathrm{V}$ of $\mathrm{y}$ such that

$$
\mathrm{U} * \mathrm{~V}^{\leftarrow} \subset \mathrm{W}
$$




\section{SEMI QUOTIENT MAPPINGS}

Definition 3.1. A mapping

$$
\phi: \mathrm{K} \rightarrow \mathrm{M} \text {, }
$$

where $K$ and $M$ are spaces is semi quotient provided a subset $E$ of $M$ is open in $M$ if and only if $\phi^{\leftarrow}(E)$ is semi open in $K$.

The differences in mappings of semi quotient, semi continuous and the quotient are illustrated below:

Example 3.1. Let

$$
\mathrm{K}=\mathrm{M}=\{1,2,3\}
$$

and let

$$
\tau_{\mathrm{K}}=\{\emptyset,\{1\},\{1,2\}, \mathrm{K}\}
$$

and

$$
\tau_{\mathrm{M}}=\{\emptyset,\{1,2\}, \mathrm{M}\}
$$

be topologies on $K$ and $M$ respectively.

Suppose

$$
\phi: \mathrm{K} \rightarrow \mathrm{M}
$$

is a mapping defined by

$$
\phi(\alpha)=\alpha, \alpha \in \mathrm{K} .
$$

Since

$$
\tau_{\mathrm{M}} \subset \tau_{\mathrm{K}},
$$

this mapping $\phi$ is semi continuous but not semi quotient because $\{1\}$ is not open in $M$, where as $\phi^{\leftarrow}(\{1\})$ is semi open in $K$.

Example 3.2. Let

$$
\begin{gathered}
\mathrm{K}=\{1,2,3,4\}, \mathrm{M}=\{\mathrm{a}, \mathrm{b}\}, \\
\tau_{\mathrm{K}}=\{\emptyset,\{2\},\{3\},\{2,3\}, \mathrm{K}\}, \tau_{\mathrm{M}}=\{\emptyset,\{\mathrm{b}\}, \mathrm{M}\} .
\end{gathered}
$$

Suppose also

$$
\phi: \mathrm{K} \rightarrow \mathrm{M} \text { by } ; \phi(4)=\phi(3)=\phi(2)=\mathrm{b} ; \phi(1)=\mathrm{a} .
$$

The mapping $\phi$ is neither continuous nor quotient but it is semi quotient because $\{b\}$ is open in $M$ but $\phi^{\leftarrow}(\{b\})=\{2,3,4\}$ is not open in $K$, that is it is not continuous. On the other hand it is semi quotient because the proper subset $\{b\} \in \tau_{M}, \phi^{\leftarrow}(\{b\})=$ $\{2,3,4\}$ is semi open in $K$.

Construction: [10] Suppose $K$ is a topological space and $M$ is a set. Suppose

$$
\phi: K \rightarrow M \text { is a mapping }
$$

and

$$
s \tau_{Q}:=\left\{E \subset M: \phi^{\leftarrow}(E) \in \mathrm{SO}(K)\right\},
$$

called the semi quotient generalized topology. But $s \tau_{Q}$ may not be a topology on $M$ 13. It appears that if $K$ is extremally disconnected, then the intersection of two semi open sets is semi open [9]. Obviously the form $s \tau_{Q}$ is the finer(stronger) than the topology $\sigma$ on $M$ :

is semi continuous. In deed,

$$
\phi: K \rightarrow(M, \sigma)
$$




$$
\phi: K \rightarrow\left(M, s \tau_{Q}\right)
$$

is a quotient mapping [11] in the present. In our coming example, we will see the relation between the spaces $(M, \sigma)$ and $\left(M, s \tau_{Q}\right)$.

Now the special case, suppose $\rho$ is an equivalence relation on $K$. Suppose

$$
p: K \rightarrow K / \rho
$$

is a projection from $K$ to the set $K / \rho: \forall \alpha$ in $K, p$ maps $\alpha$ to $\rho(\alpha)$. The generalized topology $s \tau_{Q}$ on $K / \rho$, where $K$ is extremally disconnected with, the mapping $p$ by forced semi continuous, is semi quotient. This important construction can be applied to topologized groups. We see the interesting example below [10] where a quotient topology and the semi quotient topology generated by the same mapping are different.

Example 3.3. Set

$$
\mathrm{K}=\{1,2,3,4,5\}
$$

with topology

$$
\begin{gathered}
\tau=\{\emptyset,\{1\},\{2\},\{3\},\{4\},\{1,2\},\{1,3\},\{1,4\},\{2,3\},\{2,4\},\{3,4\}, \\
\{1,2,3\},\{1,2,4\},\{1,3,4\},\{2,3,4\},\{1,2,3,4\}, K\}
\end{gathered}
$$

and the collection of semi open sets is

$$
\begin{gathered}
S O(K)=\{\emptyset,\{1\},\{2\},\{3\},\{4\},\{1,2\},\{1,3\},\{1,4\},\{1,5\}, \\
\{2,3\},\{2,4\},\{2,5\},\{3,4\},\{3,5\},\{4,5\},\{1,2,3\},\{1,2,4\}, \\
\{1,2,5\},\{1,3,5\},\{1,4,5\},\{2,3,5\},\{2,4,5\},\{3,4,5\},\{1,3,4\},
\end{gathered}
$$

$\{2,3,4\},\{1,2,3,4\},\{1,2,4,5\},\{1,2,3,5\},\{1,3,4,5\},\{2,3,4,5\},\{1,2,3,4,5\}\}$.

We define a relation $N$ on $K$ by $\alpha N \gamma \Longleftrightarrow \alpha+\gamma$ is even. Then, $N=\{(1,1),(1,3),(1,5),(2,2),(2,4),(3,1),(3,3),(3,5),(4,2),(4,4),(5,1),(5,3),(5,5)\}$ forms an equivalence relation, and

$$
\mathrm{K} / \mathrm{N}=\{\mathrm{N}(1), \mathrm{N}(2)\}=\{\{1,3,5\},\{2,4\}\} .
$$

Suppose

$$
\mathrm{p}: \mathrm{K} \rightarrow \mathrm{K} / \mathrm{N}
$$

is a canonical projection. So,

$$
\mathrm{p}^{\leftarrow}(\mathrm{N}(1))=\{1,3,5\} \in \mathrm{SO}(\mathrm{K})
$$

and

$$
\mathrm{p}^{\leftarrow}(\mathrm{N}(2))=\{2,4\} \in \mathrm{SO}(\mathrm{K})
$$

so that

$$
s \tau_{Q}=\{\emptyset, K / N,\{N(1)\},\{N(2)\}\}
$$

is the semi quotient topology on $K / N$. But, quotient topology on $K / N$ is

$$
\tau_{Q}=\{\emptyset, K / N,\{N(2)\}\} .
$$

because here

is not open in $K$.

$$
\mathrm{p}^{\leftarrow}(\mathrm{N}(1))=\{1,3,5\}
$$


Lemma 3.1. 1] If $(J, *, \tau)$ is an $s$-topological group, then $A$ is semi open in $J$ if and only if $A^{\leftarrow}$ is semi open in $J$; and if $A$ is semi open in $J$, and $B \subset J$, then $A * B$ and $B * A$ are semi open in $J$.

Lemma 3.2. 14] If $(J, *, \tau)$ is an irresolute topological group, then $A$ is semi open in $J$ if and only if $A^{\leftarrow}$ is semi open in $J$; and if $A$ is semi open in $J$, and $B \subset J$, then $A * B$ and $B * A$ are semi open in $J$.

\section{SEMI QUOTIENT MAPPINGS AND SPACES PROPERTIES}

In the present sight we will use the concept of $s \tau_{Q}$ discussed in the previous section establishing some properties.

Theorem 4.1. If $L$ is a closed subgroup of an extremally disconnected irresolute topological group $(J, *, \tau)$, and $n_{1} \in J$, then $\lambda_{n_{1}}$ is a semi-isomorphism and also

$$
p \circ \ell_{n_{1}}=\lambda_{n_{1}} \circ p .
$$

Proof. The mappings $p: J \rightarrow J / L$ and $\lambda_{n_{1}}: J / L \rightarrow J / L$ are defined by $p(x)=x * L$ and $\lambda_{n_{1}}(x * L)=n_{1} * x * L$ respectively. We see the properties of $\lambda_{n_{1}}$ as under: $\lambda_{n_{1}}$ is well defined Let $x * L=y * L$. This implies $n_{1} * x * L=n_{1} * y * L$. This implies that $\lambda_{n_{1}}(x * L)=$ $\lambda_{n_{1}}(y * L)$.

$\lambda_{n_{1}}$ is injective

Let $\lambda_{n_{1}}(x * L)=\lambda_{n_{1}}(y * L)$. This implies that $n_{1} * x * L=n_{1} * y * L$. By left cancelation law, we get $x * L=y * L$.

$\lambda_{n_{1}}$ is surjective

For every $n_{1} * x * L$ in the range of $\lambda_{n_{1}}$, there exists $x * L$ in the domain of $\lambda_{n_{1}}$ such that $\lambda_{n_{1}}(x * L)=n_{1} * x * L$.

$\lambda_{n_{1}}$ is homomorphism

Since $p(x * y)=x * y * L=x * L * y * L=p(x) * p(y)$. Therefore, $\lambda_{n_{1}}$ is homomorphism. We have to show that

$$
p \circ \ell_{n_{1}}=\lambda_{n_{1}} \circ p .
$$

In fact, $\forall \alpha \in J$ we get,

$$
\left(p \circ \ell_{n_{1}}\right)(\alpha)=p\left(n_{1} * \alpha\right)=\left(n_{1} * \alpha\right) * L=n_{1} *(\alpha * L)=\lambda_{n_{1}}(p(\alpha))=\left(\lambda_{n_{1}} \circ p\right)(\alpha) .
$$

Now remaining is to show that $\lambda_{n_{1}}$ is pre semi open and irresolute. It is evident from the followings. Suppose

$$
\alpha * L \in J / L .
$$

For every semi open set $U$ of $e_{J}$,

$$
p(\alpha * U * L)
$$

is a semi open set of

$$
\alpha * L \text { in } J / L
$$

In the same strategy,

$$
p\left(n_{1} * \alpha * U * L\right)
$$

is a semi open set of

$$
n_{1} * \alpha * L
$$

in $J / L$. 
Theorem 4.2. If $\left(J, *, \tau_{J}\right)$ is an $s$ - topological group, then $\left(J / N, *, s \tau_{Q}\right)$ is extremally disconnected s-topological group, where $N$ is an invariant subgroup of $J$

Proof. Since $\left(J, *, \tau_{J}\right)$ is an $s$ - topological group, then by definition for every open neighbourhood $W$ of $x * y^{\leftarrow}$, there exist semi open neighbourhoods $U$ of $x$ and $V$ of $y$ such that $U * V \subset W$. By Lemma 3.1. $U * N$ and $V * N$ are respective semi open neighbourhoods of $x * N$ and $y * N$. By using hypothesis, $U * V * N \subset W * N$. This implies that $(U * N) *(V * N) \subset W * N$. Hence $\left(J / N, *, s \tau_{Q}\right)$ is extremally disconnected $s$-topological group.

Theorem 4.3. Let $N$ be an invariant subgroup and $\zeta: J / N \rightarrow H / N$ be an $S$-isomorphism of quasi s-topological groups. If $\zeta$ is semi continuous at the neutral element of the domain, then it is semi continuous at the domain.

Proof. Let $x * N \in J / N$ and $W * N$ be an open neighbourhood of $y * N=\zeta(x * N)$ in $H / N$. Then by semi continuity of left translation in $H / N$, there exists a semi open neighbourhood $V * N$ of the neutral element $N$ of $H / N$ such that (i) $\ell_{y * N}(V * N)=$ $y * N * V * N \subset W * N$. By the hypothesis, $\zeta$ is semi continuous at $e_{J} * N=N$ implies (ii) $\zeta(U * N) \subset N * N$, for some semi open neighbourhood $U * N$ of $e_{J} * N$. Also $\ell_{x * N}: J / N \rightarrow J / N$ is an $s$-open mapping, the set $\ell_{x * N}(U * N)=x * N * U * N$ is semi open in $J / N$. Hence $\zeta(x * N * U * N)=y * N *(U * N) \subset y * N * V * N \subset W * N$ (by ii and i). Thus $\zeta$ is semi continuous at $J / N$.

Theorem 4.4. Let $\left(J / N, *, s \tau_{J}\right)$ be an extremally disconnected $s$-topological group and $\left(H / N, *, s \tau_{H}\right)$ be an extremally disconnected quasi $s$-topological group with $N$ an invariant subgroup. If $\zeta: J / N \rightarrow H / N$ is $S$-isomorphism with $\zeta\left(x^{\leftarrow}\right)=$ $(\zeta(x))^{\leftarrow}$, then $H / N$ is also extremally disconnected s-topological group.

Proof. Let $W * N=O_{h_{1} * N * h_{2}^{\leftarrow} * N}$ be an open neighbourhood of $h_{1} * N * h_{2}^{\leftarrow} * N$, where $h_{1}, h_{2} \in H$. Then by semi continuity of $\zeta, \zeta^{\leftarrow}(W * N)=\zeta^{\leftarrow}\left(O_{h_{1} * N * h_{2}^{\leftarrow} * N}\right)$ is semi open neighbourhood in $J / N$. Also $\zeta$ is bijective, $\zeta\left(g_{1}\right)=h_{1}$ and $\zeta\left(g_{2}\right)=h_{2}$, where $g_{1}, g_{2} \in J$. This implies $g_{1}=\zeta^{\leftarrow}\left(h_{1}\right), g_{2}=\zeta^{\leftarrow}\left(h_{2}\right)$. Since $J / N$ is extremally disconnected $s$-topological group, there are semi open neighbourhoods $M_{g_{1} * N}$ and $M_{g_{2} * N}$ such that $M_{g_{1} * N} * M_{g_{2}^{\leftarrow} * N} \subset \zeta^{\leftarrow}(W * N)$. This gives that $\zeta\left(M_{g_{1} * N} *\right.$ $\left.M_{g_{2} * N}\right) \subset W * N$. By homomorphism of $\zeta$, we get $\zeta\left(M_{g_{1} * N}\right) * \zeta\left(M_{g_{2}^{\leftarrow} * N}\right) \subset W * N$. Since $\zeta$ is $s$-open, then $\zeta\left(M_{g_{1} * N}\right)$ and $\zeta\left(M_{g_{2} * N}\right)$ are semi open neighbourhoods in $H / N$. This gives that $\left(W_{h_{1} * N}\right) *\left(W_{h_{2} \leftarrow N}\right) \subset W * N$, where $\zeta\left(W_{h_{1} * N}\right)=W_{h_{1} * N}$ and $\zeta\left(M_{g_{2} * N}\right)=W_{h_{2} * N}$. That is, $\left(W_{h_{1} * N}\right) *\left(W_{h_{2} * N}\right)^{\leftarrow} \subset O_{h_{1} * N * h_{2} * N}$.

Theorem 4.5. If $L$ is a closed invariant subgroup of an extremally disconnected $s$-topological group $(J, *, \tau)$, and if $\zeta: J / L \rightarrow H / L$ is $S$-isomorphism with $\zeta\left(x^{\leftarrow}\right)=$ $(\zeta(x))^{\leftarrow}$, where $\left(J / L, *, s \tau_{J}\right)$ is an extremally disconnected s-topological group and $\left(H / L, *, s \tau_{H}\right)$ is an extremally disconnected quasi $s-$ topological group, then $\left(J / L, *, s \tau_{Q}\right)$ is an extremally disconnected s-topological group.

Proof. Suppose the left translations

$$
l_{g}: J \rightarrow J \text { and } l_{p(g)}: J / L \rightarrow J / L
$$

by $g \in J$, and $p(g) \in J / L$, and the inverse mappings $i$ and $i^{\prime}$ respectively. $\forall \alpha \in J$, we get

$$
\left(p \circ l_{g}\right)(\alpha)=g * \alpha * L=(g * L) *(\alpha * L)(=m(g * L, \alpha * L))=\left(l_{p(g)} \circ p\right)(\alpha)
$$


and

$$
(p \circ i)(\alpha)=\alpha^{-1} * L=\left(i^{\prime} \circ p\right)(\alpha) .
$$

We see following commutative figures:

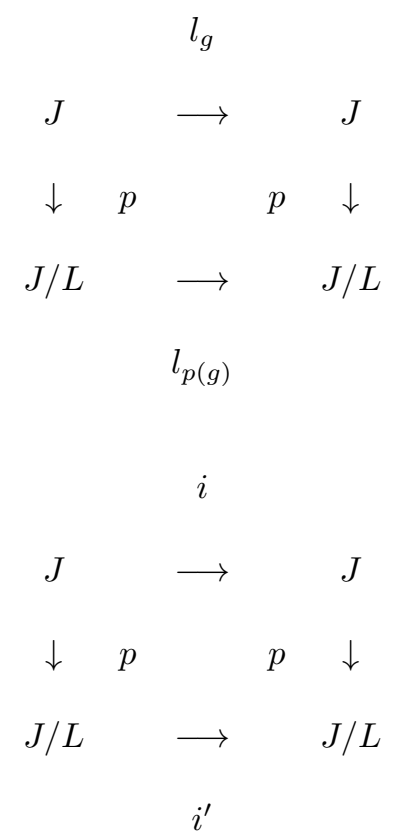

Here $p$ is semi continuous, and the left translation

$$
l_{g}: J \rightarrow J
$$

$l_{g}(\alpha)=g * \alpha, \forall \alpha \in J$.

Claim: left translation $l_{g}$ and the inverse mapping $i$ are irresolute.

If $W$ is a semi open of $g * \alpha$ from range $J, g^{-1} * W$ is a semi open of domain element $\alpha$ satisfying

$$
l_{g}\left(g^{-1} * W\right)=W .
$$

Suppose $W$ is a semi open in $i(\alpha)$. By Lemma 3.2 [14], $W^{-1}$ is a semi open set of the domain element $\alpha$ satisfying

$$
i\left(W^{-1}\right)=W
$$

This gives that the left translation $l_{p(g)}$ and the inverse mapping $i^{\prime}$ must be semi continuous. This gives that $J / L$ is an extremally disconnected quasi $s$-topological group. Thus by Theorem 4.4, we have the required result.

\section{REFERENCES}

[1] E. Bohn, Semi-topological groups, The American Mathematical Monthly, 72(9)(1965), 996998.

[2] M. S. Bosan and M. D. Khan, On quasi irresolute and semi Irr-topological groups, Afinidad, 80(574)(2014), 1241-1252. 
[3] M. S. Bosan, M. D. Khan and L. DR. Kočinac, On s-topological groups, Mathematica Moravica, 80(2)(2014), 35-44.

[4] S. G. Crossley, SK. Hildebrand, Semi-topological properties, Fundamenta Mathematicae, 74(3)(1972), 233-254

[5] M. D. Khan and M. S. Bosan, A note on s-topological groups, Life Sci. J., 11(2014), 370-374.

[6] JP. Lee, On semi-homeomorphisms, International Journal of Mathematics and Mathematical Sciences, 13(1990), 129-134.

[7] N. Levine, Semi-open sets and semi-continuity in topological spaces, The American Mathematical Monthly, 70(1)(1963), 36-41.

[8] James R Munkres, Topology, Prentice Hall Upper Saddle River, NJ, 2000.

[9] O. Njastad, On some classes of nearly open sets, Pacific Journal of Mathematics, 15(3)(1965), 961-970.

[10] R. Noreen, M. S. Bosan and M. D. Khan, Semi-quotient mappings and spaces, Open Mathematics, 14(1)(2016), 1014-1022.

[11] C. W. Patty, Foundations of topology, Jones \& Bartlett Learning, 2009.

[12] D. JS. Robinson, A course in the theory of groups, vol. 80, Springer Science \& Business Media, 2012

[13] R. Shen, Remarks on products of generalized topologies, Acta Mathematica Hungarica, 124(4)(2009), 363-369.

[14] A. Siab, L. DR. Kočinac and M. D. Khan, Irresolute-topological groups, Mathematica Moravica, 19(1)(2015), 73-80.

Department of Mathematics,

Khwaja Fareed University of Engineering and Information Technology, Rahim Yar Khan, 64200, Pakistan, OrCid ID: 0000-0002-9433-7960

Email address: siddiquebosan1966@gmail.com 